\title{
Controlling the model sign problem via the path optimization method: Monte Carlo approach to a QCD effective model with Polyakov loop
}

\author{
Kouji Kashiwa, ${ }^{1,2, *}$ Yuto Mori, ${ }^{3, \dagger}$ and Akira Ohnishi ${ }^{2, \$}$ \\ ${ }^{1}$ Fukuoka Institute of Technology, Wajiro, Fukuoka 811-0295, Japan \\ ${ }^{2}$ Yukawa Institute for Theoretical Physics, Kyoto University, Kyoto 606-8502, Japan \\ ${ }^{3}$ Department of Physics, Faculty of Science, Kyoto University, Kyoto 606-8502, Japan
}

(Received 3 June 2018; published 24 January 2019)

\begin{abstract}
We apply the path optimization method to a QCD effective model with the Polyakov loop at finite density to circumvent the model sign problem. The Polyakov-loop extended Nambu-Jona-Lasinio model is employed as the typical QCD effective model and then the hybrid Monte Carlo method is used to perform the path integration. To control the sign problem, the path optimization method is used with complexification of temporal gluon fields to modify the integral path in the complex space. We show that the average phase factor is well improved on the modified integral-path compared with that on the original one. This indicates that the complexification of temporal gluon fields may be enough to control the sign problem of QCD in the path optimization method.
\end{abstract}

DOI: 10.1103/PhysRevD.99.014033

\section{INTRODUCTION}

Investigation of the phase structure of quantum chromodynamics (QCD) is one of the important subjects in the study of the hot and dense QCD matter. If we directly obtain the phase diagram at finite temperature $(T)$ and density from the first-principles calculation such as the lattice QCD simulation, there are no fog in the exploration. Lattice QCD, however, has the sign problem at finite real chemical potential $(\mu)$ and then we cannot obtain the reliable results at finite density. To circumvent the sign problem, several methods have been proposed such as the Taylor expansion method [1-3], the reweighting method $[4,5]$, the analytic continuation method [6-8], the canonical approach [9-13] and so on. However, we cannot go beyond the $\mu / T \sim 1$ line by using those methods; e.g., see Ref. [14].

Recently, new ideas have been applied to attack the sign problem such as the complex Langevin method $[15,16]$ and the Lefschetz-thimble method [17-19]. Both methods are based on the complexification of dynamical variables. The complex Langevin method is based on the stochastic quantization and thus it does not have the sign problem, in principle. In the Lefschetz-thimble method, one should

\footnotetext{
*kouji.kashiwa@yukawa.kyoto-u.ac.jp

†mori@ruby.scphys.kyoto-u.ac.jp

ohnishi@yukawa.kyoto-u.ac.jp
}

Published by the American Physical Society under the terms of the Creative Commons Attribution 4.0 International license. Further distribution of this work must maintain attribution to the author(s) and the published article's title, journal citation, and DOI. Funded by SCOAP. solve flow equations to construct the new integral path which is corresponding to the steepest descent trajectory; this trajectory is so called the Lefschetz thimble. Unfortunately, these methods have some serious problems and thus it is difficult to apply it to QCD at high density: In the complex Langevin method, there is the possibility that it is converged to wrong results due to the excursion and singular problems [20,21]. In comparison, the Lefschetz thimble method has the global sign problem when two or more Lefschetz thimbles contribute to the path integral and then there is the serious cancellation between them; e.g., see Ref. [19]. In addition, we should evaluate the Jacobian induced by the modification of the integral path and it leads the serious increase of the numerical cost. Furthermore, the Jacobian induces the residual sign problem because the oscillation of the Boltzmann weight arises. Also, we may face the problem to draw thimbles when the classical or the effective action has singular points [22]. Recently, some attempts to extend the complex Langevin method and the Lefschetz thimble method have been proposed; the unification approach of both methods [23], the modification method [24], the extension of the thimble regularization $[25,26]$, the dynamical stabilization [27], the path optimization [28-31] and the sign-optimized manifold construction [32]. In this study, we concentrate on the path optimization method (POM).

In Ref. [28], we have proposed the new method which we call the path optimization method motivated by the Lefschetz thimble method. The path optimization method is strongly improved in Ref. [29] by introducing the feedforward neural network to optimize the modified integral path. In this method, we first complexify the variables of 
integration as in the Lefschetz thimble method. Then, the modified integral path is constructed to minimize the cost function which reflects the seriousness of the sign problem. Therefore, we can treat the sign problem as the optimization problem. The sign problem in the simple one-dimensional integration $[28,30]$ and the complex $\lambda \phi^{4}$ theory [29] are found to be under controlled. Unfortunately, the numerical cost of the path optimization method is still heavy and thus we cannot apply it to QCD yet, but this method has large extensibility compared with the Lefschetz thimble method and thus we can still dream to apply it to QCD in the future.

Because of several difficulties in QCD as mentioned above, QCD effective models have been widely used to investigate the QCD phase structure because such effective model is much easier than original QCD. The Polyakov-loop extended Nambu-Jona-Lasinio (PNJL) model [33-35] is one of the famous and powerful effective models. Unfortunately, the PNJL model has the model sign problem [36,37] even in the mean-field approximation because one particular global minimum perfectly dominate the path integration and then the thermodynamic potential can be complex. It should be noted that this complex nature of the thermodynamic potential is not related with instabilities; the correct thermodynamic potential should be real.

In this study, we consider the PNJL model and the path optimization method to circumvent the model sign problem. This article is organized as follows. In Secs. II and III, we explain the formulation of the PNJL model and the path optimization method, respectively. The numerical results are shown in Sec. IV. Section V is devoted to summary.

\section{FORMULATION}

In this work, we employ the PNJL model as the QCD effective model. The PNJL model can describe the chiral symmetry breaking/restoration and the approximate confinement-deconfinement transition. Also, it can well reproduce QCD properties at finite imaginary chemical potential which is a big advantage from the viewpoint of the topologically determined confinement-deconfinement transition [38-40]. The following formulation and the computation of the Monte-Carlo PNJL model is based on Ref. [41].

\section{A. Polyakov-loop extended Nambu-Jona-Lasinio model}

The Euclidean action of the two-flavor PNJL model is

$$
\begin{aligned}
\Gamma_{\mathrm{PNJL}}= & \int d^{4} x_{\mathrm{E}}\left[\bar{q}\left(-i \not D+m_{0}-\mu \gamma_{0}\right) q\right. \\
& \left.-G\left\{(\bar{q} q)^{2}+\left(\bar{q} i \vec{\tau} \gamma_{5} q\right)^{2}\right\}\right]-\beta V \mathcal{V}_{\Phi}(\Phi, \bar{\Phi}),
\end{aligned}
$$

where $\beta=1 / T, V$ is the three-dimensional spatial volume, $q$ denotes the two-flavor quark-fields, $m_{0}$ does the current quark mass, $D_{\nu}=\partial_{\nu}-i A_{\nu} \delta_{\nu 4}, G$ is the coupling constant of the four-fermi interaction, $\Phi(\bar{\Phi})$ means the Polyakov loop (its conjugate) and $\mathcal{V}_{\Phi}$ expresses the gluonic contribution.

The (effective) action which is used in the present Monte-Carlo calculation is $S=\beta V \mathcal{V}$ where $\mathcal{V}$ is the effective potential and the grand-canonical partition function then becomes

$$
\mathcal{Z}=\int \prod_{\mathbf{k}} d z_{k} e^{-S\left[z_{k}\right]}
$$

where $z_{\mathbf{k}}$ means the dynamical variables in the momentum space. In the present study, we truncate the auxiliary fields to $\mathbf{k}=0$ components and thus our Monte-Carlo result is matched with the mean-field approximation in the infinite volume limit. This truncation, the homogeneous field ansatz, is also used in the previous work of the Monte Carlo PNJL model with $\mu=0$ in Ref. [41].

With the homogeneous auxiliary-field ansatz after the Hubbard-Stratonovich transformation, the effective action is simplified as $\Gamma=\beta V \mathcal{V}$. Then, the effective potential is expressed as

$$
\mathcal{V}=\mathcal{V}_{\mathrm{NJL}}+\mathcal{V}_{\Phi}
$$

where $\mathcal{V}_{\mathrm{NJL}}$ is the contributions of the NJL part. In the actual calculation, we employ the Polyakov gauge, $\partial_{4} A_{4}=0$.

The actual form of $\mathcal{V}_{\mathrm{NJL}}$ becomes

$$
\begin{aligned}
\mathcal{V}_{\mathrm{NJL}}= & -2 N_{\mathrm{f}} \int_{\Lambda} \frac{d^{3} p}{(2 \pi)^{3}}\left[N_{\mathrm{c}} E_{\mathbf{p}}-N_{\mathrm{c}} \sqrt{\mathbf{p}^{2}+m_{0}^{2}}\right. \\
& \left.+T \ln \left(f^{-} f^{+}\right)\right]+G\left(\sigma^{2}+\vec{\pi}^{2}\right),
\end{aligned}
$$

where $N_{\mathrm{f}}\left(N_{\mathrm{c}}=3\right)$ is the number of flavor (color), $\Lambda$ is the three-dimensional momentum cutoff and

$$
\begin{aligned}
& f^{-}=1+3\left(\Phi+\bar{\Phi} e^{-\beta E_{\mathbf{p}}^{-}}\right) e^{-\beta E_{\mathbf{p}}^{-}}+e^{-3 \beta E_{\mathbf{p}}^{-}} \\
& f^{+}=1+3\left(\bar{\Phi}+\Phi e^{-\beta E_{\mathbf{p}}^{+}}\right) e^{-\beta E_{\mathbf{p}}^{+}}+e^{-3 \beta E_{\mathbf{p}}^{+}}
\end{aligned}
$$

with $\quad E_{\mathbf{p}}^{\mp}=E_{\mathbf{p}} \mp \mu=\sqrt{\varepsilon_{\mathbf{p}}^{2}+2 N^{+} N^{-}} \mp \mu$ and $\varepsilon_{\mathbf{p}}=$ $\sqrt{\mathbf{p}^{2}+M^{2}+N^{2}}$. In the present calculation, we keep the momentum cutoff in the thermal contributions. The auxiliary fields are redefined as

$$
M=m_{0}-2 G \sigma, \quad N=-2 G \pi^{0}, \quad N^{ \pm}=-2 G \pi^{ \pm} .
$$

with $\pi^{0}=\pi_{3}$ and $\pi^{ \pm}=\left(\pi_{1} \pm i \pi_{2}\right) / \sqrt{2}$. The unit of $M$ and $\sigma$ are $[\mathrm{GeV}]$ and $\left[\mathrm{GeV}^{3}\right]$ and thus $G$ is $\left[\mathrm{GeV}^{-2}\right]$. The functional form of the Polyakov loop and its conjugate are 


$$
\begin{aligned}
& \Phi=\frac{1}{N_{\mathrm{c}}} \operatorname{tr}_{c}\left[e^{i \beta A_{4}}\right]=\frac{1}{N_{\mathrm{c}}}\left[e^{i \beta \phi_{1}}+e^{i \beta \phi_{2}}+e^{i \beta \phi_{3}}\right], \\
& \bar{\Phi}=\frac{1}{N_{\mathrm{c}}}\left[e^{-i \beta \phi_{1}}+e^{-i \beta \phi_{2}}+e^{-i \beta \phi_{3}}\right]
\end{aligned}
$$

where

$$
\begin{aligned}
& \phi_{1}=A_{3}+\frac{1}{\sqrt{3}} A_{8}, \quad \phi_{2}=-A_{3}+\frac{1}{\sqrt{3}} A_{8} \\
& \phi_{3}=-\left(\phi_{1}+\phi_{2}\right)=-\frac{2}{\sqrt{3}} A_{8},
\end{aligned}
$$

and then $A_{4}$ are diagonalized because we use the Polyakov gauge; $A_{4}=\operatorname{diag}\left(A_{3}+A_{8} / \sqrt{3},-A_{3}+A_{8} / \sqrt{3},-2 A_{8} / \sqrt{3}\right)$.

In this paper, we choose the logarithmic Polyakov-loop potential [42] as the gluonic contribution;

$$
\frac{\mathcal{V}_{\Phi}}{T^{4}}=-\frac{1}{2} a_{T} \bar{\Phi} \Phi+b_{T} \ln (h)
$$

where

$$
h=1-6 \bar{\Phi} \Phi+4\left(\bar{\Phi}^{3}+\Phi^{3}\right)-3(\bar{\Phi} \Phi)^{2},
$$

with

$a_{T}=a_{0}+a_{1}\left(\frac{T_{0}}{T}\right)+a_{2}\left(\frac{T_{0}}{T}\right)^{2}, \quad b_{T}=b_{3}\left(\frac{T_{0}}{T}\right)^{3}$.

The parameters should be set to reproduce the lattice QCD data in the pure gauge limit. In the present calculation, we complexify $A_{3}$ and $A_{8}$ to weaken the sign problem and then the imaginary part of $A_{3}$ and $A_{8}$ are fixed via Eq. (14); details are shown in Sec. III.

The present Polyakov-loop potential may induce the singularities such as the logarithmic cut. One of the promising attempts to deal with this problem is the modification of the Polyakov-loop potential. The second term of the Polyakov-loop potential appears as $V T_{0}^{3} b_{3} \times$ $\ln (h)$ in the Boltzmann weight. Therefore, when $V T_{0}^{3} b_{3}$ becomes the natural number, the singularity does not matter. In the present case, $V T_{0}^{3} b_{3}$ is not the natural number, but it depends on the model details. It is known that there is another form of the Polyakov-loop potential that is the polynomial form [35] which can also reproduce the lattice QCD data in the pure gauge limit; this model does not have the logarithmic singularity. Nevertheless, it should be noted that the path optimization method can reproduce the correct result with the logarithmic Polyakovloop potential as shown later. Therefore, the path optimization method can practically work well in the present setup.

\section{B. System volume and parameters}

In this study, we follow the lattice formalism and thus $V$ can be expressed [41] as

$$
V=N_{s}^{3} a^{3}=\frac{N_{s}^{3}}{T^{3} N_{t}}=\frac{k}{T^{3}},
$$

where $N_{s}\left(N_{t}\right)$ are the number of spatial (temporal) lattice sites and $a$ is the lattice spacing. Then, $V$ should depend on the temperature. In this article, we use the homogeneous ansatz and thus our Monte Carlo simulation reaches the mean-field results in the infinite volume limit. The finite size effect is just jumped into the game via $V$ on the Boltzmann weight in the present calculation and then the effective potential itself does not have explicit finite size effect. This treatment is perfectly matched with that used in Ref. [41]. In principle, we must consider the lattice regularization in order to introduce the finite size effects. However, such inconsistency becomes smaller and smaller when the system volume becomes larger and larger and thus it is a minor problem in this study. Full simulation of the lattice PNJL model is our future work.

The present PNJL model has three parameters, $G, m_{0}$ and $\Lambda$ in the NJL part. The actual values of the parameters are taken from Ref. [43]; $m_{0}=5.5 \mathrm{MeV}, G=$ $5.498 \mathrm{GeV}^{-2}$ and $\Lambda=631.5 \mathrm{MeV}$. The parameters in the Polyakov-loop potential is taken from Ref. [42];

$a_{0}=3.51, \quad a_{1}=-2.47, \quad a_{2}=15.2, \quad b_{3}=-1.75$,

with $T_{0}=270 \mathrm{MeV}$.

\section{PATH OPTIMIZATION METHOD}

To deal with the model sign problem appearing at finite density, we here introduce the path optimization method.

\section{A. Introduction to POM}

In the path optimization method, we start from the complexification of the variables of the integration, $x_{i} \in \mathbb{R} \rightarrow z_{i} \in \mathbb{C}$ where $i=1, \ldots, n$ with the dimension of integration $n$. To construct the new integral path in the complex plane, we prepare the cost function which should be related with the seriousness of the sign problem. The functional form of the new integral path is represented by using the feedforward neural network with some parameters which are optimized via the minimization of the cost function. Since the neural network even with the mono hidden-layer can approximate any kind of continuous function on the compact subset as long as we can use sufficient number of units in the layer $[44,45]$, the neural network seems to be suitable for the path optimization method. Details are shown in Ref. [28].

In the path optimization method, we represent $z_{i}$ by using the parametric quantity $(t)$ as 


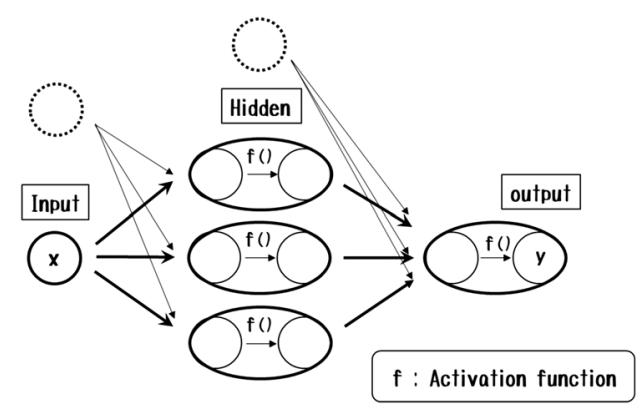

FIG. 1. The schematic figure of the feedforward neural network used in this study for the case with the single input and output. The dotted circles mean the bias and the thick arrows indicate the weight. In the final step, we calculate Eq. (14) to obtain the modified integral-path.

$$
z_{i}(t)=t_{i}+i\left[w_{i} f_{i}(t)+b_{i}\right],
$$

where $w$ and $b$ are parameters. This function means $\operatorname{Re}\left(z_{i}\right) \rightarrow \operatorname{Im}\left(z_{i}\right)$. Parameters $w, b$ and also parameters in $f$ are determined by using the back-propagation algorithm [46]. Figure 1 shows the schematic figure of the neural network used in this study. In the back-propagation, we choose tanh for the activation function. In the following, we represents parameters in the neural network as $c=\left\{c_{i}\right\}$. Details are shown in Refs. [28,29]. It should be noted that the path optimization method leads the same results with the original theory because of the Cauchy(-Poincare)'s theorem as long as the integral path does not go across singular points, $J e^{-\Gamma}=0$. In the complex Langevin method, singular points induce the problem because it leads the singular drift term in the Langevin-time evolution, the path optimization method can care for it.

In this study, we complexify $A_{3}$ and $A_{8}$ and then $\sigma$ and $\vec{\pi}$ are still treated as real variables. Since it is known that the model sign problem can be resolved by the complexification of the temporal gluon fields in the Lefschetzthimble method [37], our treatment should work. In addition, such treatment is consistent with the calculation which imposes the $\mathcal{C K}$ symmetry on the fermion determinant at finite density [47,48] where $\mathcal{C}$ and $\mathcal{K}$ denote the charge conjugation and the complex conjugation, respectively. Unfortunately, the Lefschetz-thimble method is difficult to apply to the PNJL model because we should solve flow equations and it sometimes encounter singular points of the effective potential. The NJL model with the repulsive vector-type interaction is a typical example [22]; the repulsive vector-type interaction induces the auxiliary field, $\omega_{4}=-\bar{q} i \gamma_{0} q$, and it acts similarly to the $U(1)$ gauge field in the Dirac determinant, $\operatorname{Det} \mathcal{D}(\mu)$ where $\mathcal{D}(\mu)$ is the Dirac operator. The origin of the sign problem is induced via the combination of $\mu-i \omega_{4}$ in $\mathcal{D}(\mu)$ and the origin is perfectly same with the case with the gluon field which is the $S U(3)$ gauge field. Of course, $S U(3)$ is a more complicated group than the $U(1)$ group and thus the sign problem is more serious in the $S U(3)$ gauge theory, but similar tendency should be there. In comparison, the path optimization method can avoid the problem and thus it is suitable for the PNJL model analysis. It should be noted that we may expect that the sign problem in QCD can be weaken via the complexification of $A_{3}$ and $A_{8}$ because the direct origin of the sign problem in QCD is the combination of the temporal component of the gluon field and the chemical potential in the Dirac determinant. It is perfectly the same with the PNJL model. In the PNJL model, the origin of the sign problem is clearly stated in Ref. [49] and $A_{8}$ is the main term which induces the sign problem in the PNJL model. The other components of the gluon field may strengthen the sign problem in QCD, but these are indirect origins of the sign problem. If it is strong enough accidentally, we should complexify all of the degree of freedom.

It should be noted that the present path optimization with the machine learning is unsupervised learning because we do not need teacher data to obtain optimized parameters in the neural network. We try to increase the average phase factor compared with that in the previous optimization step. The one attempt to introduce the supervised learning to the study of the sign problem has been done in Ref. [50] based on the generalized Lefschetz thimble method [51].

\section{B. Optimization process}

We use the following cost function in the calculation;

$$
\begin{aligned}
\mathcal{F}[z(t)] & =\frac{1}{2} \int d^{n} t\left|e^{i \theta(t)}-e^{i \theta_{0}}\right|^{2} \times\left|J(t) e^{-\Gamma(z(t))}\right| \\
& =\int d^{n} t\left|J(t) e^{-\Gamma(z(t))}\right|-\left|\int d^{n} t J(t) e^{-\Gamma(z(t))}\right|,
\end{aligned}
$$

where

$$
\begin{aligned}
& \theta(t)=\arg \left(J(t) e^{-\Gamma(z(t))}\right), \quad \theta_{0}=\arg (\mathcal{Z}), \\
& J(t)=\operatorname{det}\left(\frac{\partial z_{i}}{\partial t_{j}}\right),
\end{aligned}
$$

with the partition function $\mathcal{Z}$. In the equation, $z_{i}$ represent complexified $A_{3}$ and $A_{8}$ and also the real $\sigma$ and $\vec{\pi}$. Thus, we have $6\left(\sigma, \pi^{0}, \pi^{ \pm}, \operatorname{Re} A_{3}, \operatorname{Re} A_{8}\right)+2\left(\operatorname{Im} A_{3}, \operatorname{Im} A_{8}\right)$ dynamical variables where latter two variables are induced via the complexification and fixed via Eq. (14). If we wish to take care of the periodicity of the effective potential for $\operatorname{Re} A_{3}$ and $\operatorname{Re} A_{8}$, we should use periodic functional form for those as inputs of the neural network like as Ref. [50]. When the wide spreading of the configurations in the Monte-Carlo calculation becomes important, the periodicity issue may become serious problem from the viewpoint of the Cauchy-Poincaré's theorem. We will revisit this point in our forthcoming paper. In this study, configurations are well localized in the range $-\pi \leq \operatorname{Re} A_{3} / T \leq \pi$ and $-\pi \leq$ $\operatorname{Re} A_{8} /(T \sqrt{3}) \leq \pi$ by using the simple complexification of 
$A_{3}$ and $A_{8}$. Of course, all points on the integral path can be taken if we gather infinite number of configurations. In the case with the finite number of configurations, the configurations are localized and then the statistical sum should work. This localization sometime induces the problem if there are several relevant saddle points which is nothing but the global sign problem; if the global sign problem arises, we must consider the parallel tempering method used in the Lefschetz-thimble method $[52,53]$. In the present model, we should have the global sign problem very close to the first-order transition line, but we are working in the crossover region at present. In the NJL model, there are at least two thimbles in the case with the first-order chiral phase transition [29], but we consider $T=100 \mathrm{MeV}$ in this study and then the transition is crossover. Therefore, we can expect that the dominant thimble is only one in the present calculation. Therefore, the localization means that the present calculation practically works well. As shown later, the present calculation actually well matched with the mean-field approximation. Thus, we do not introduce the periodic form of inputs in this study. This cost function is proportional to $\left\langle e^{i \theta}\right\rangle$ and thus it reflects the seriousness of the sign problem [29]. In the physical system, $\theta_{0}$ should be 0 . If we consider $\theta_{0} \neq 0$, we can apply the path optimization method to other systems such as the complex chemical potential.

In the hybrid Monte-Carlo method, we should evaluate the expectation value of the cost function. To do this, we replace the cost function as

$$
\mathcal{F}[z(t)] \rightarrow \frac{\mathcal{F}[z(t)]}{\int d^{n} t \mathcal{P}(t)},
$$

where $\mathcal{P}$ is the appropriate probability distribution.

In the back-propagation procedure, we need the derivative of the cost function by $c_{i}$. We represent it as $d \mathcal{F}_{i}$ below. After straightforward calculations, we finally reach the expression

$$
\begin{aligned}
d \mathcal{F}_{i}(t, c)= & \left|J(t) e^{-\Gamma(z(t))}\right| \\
& \times \operatorname{Re}\left[\left(1-e^{i\left(\theta(t)-\theta_{0}\right)}\right) \frac{\partial}{\partial c_{i}} \log \left(J(t) e^{-\Gamma(z(t))}\right)\right],
\end{aligned}
$$

In the hybrid Monte-Carlo method, we rewrite Eq. (18) as similar to Eq. (17) with $\mathcal{P}$. This cost function is responsible to the alignment of the Boltzmann weight with each other on the modified integral path if the points are relevant to the path-integral.

To make our optimization easier, we employ the minibatch training. The configurations are divided as $N_{\text {config }}=$ $n N_{\text {batch }}$ where $N_{\text {batch }}$ is the batch size and then the learning is performed batch by batch. To include all updates of each batch, the parameters in the feedforward neural network is updated by replacing $d F_{i}$ by its mean-value as

$$
d \mathcal{F}_{i}(t, c) \rightarrow \frac{1}{N_{\text {batch }}} \sum_{k=1}^{N_{\text {batch }}} d \mathcal{F}^{(k)}(t, c) .
$$

In one optimization step, we update $n$-times with $N_{\text {batch }}$ configurations.

In this study, we use the simple feedforward neural network with the hidden monolayer. Input is the original integral path and output is its imaginary part. For the optimizer, we employ the Adam algorithm [54];

$$
\begin{aligned}
v_{i}^{(j+1)} & =\beta_{1} v^{(j)}+\left(1-\beta_{1}\right) d \mathcal{F}_{i}, \\
r_{i}^{(j+1)} & =\beta_{2} r^{(j)}+\left(1-\beta_{2}\right) d \mathcal{F}_{i}^{2}, \\
\hat{v}_{i}^{(j+1)} & =\frac{v_{i}^{(j+1)}}{1-\beta_{1}^{j}}, \quad \hat{r}_{i}^{(j+1)}=\frac{r_{i}^{(j+1)}}{1-\beta_{2}^{j}}, \\
c_{i}^{(j+1)} & =c_{i}^{(j)}-\frac{\eta}{\sqrt{\hat{r}_{i}^{(j+1)}}+\epsilon} \hat{v}_{i}^{(j+1)},
\end{aligned}
$$

where $j$ is the fictitious time step, $d \mathcal{F}_{i}$ means $\partial \mathcal{F} / \partial c_{i}$ and $\beta_{1}$ and $\beta_{2}$ are the smoothing factors of the exponential moving average. This algorithm is based on the AdaGrad algorithm [55] and the momentum method with preventing the learning weight decay.

\section{Simulation setup}

The number of units in the hidden layer is set to $N_{\text {unit }}=4$. For Adam algorithm, we use $\eta=0.001$, $\alpha=0.999, \beta=0.9$ and $\epsilon=10^{-8}$. In the minibatch training, we set to $N_{\text {batch }}=10$. These parameters are so called hyper parameters in the machine learning. Initial values of parameters in the neural network are prepared based on Xavier initialization [56].

In the calculation of the expectation values of operators, we have generated 80000 configurations analyzed each 50 trajectories for each optimization step. Then, the expectation values are estimated after 2 times optimization. Statistic errors are obtained by using the jackknife method where the bin number is set to 10 . The expectation value of an operator $(\mathcal{O})$ is obtained via the phase reweighting as

$$
\langle\mathcal{O}\rangle=\frac{\int d^{n} t \mathcal{O} e^{i \theta}\left|J(t) e^{-\Gamma(z(t))}\right|}{\int d^{n} t e^{i \theta}\left|J(t) e^{-\Gamma(z(t))}\right|} .
$$

In this study, we calculate the chiral condensate and the Polyakov loop.

\section{NUMERICAL RESULTS}

The $T$-dependence of the chiral condensate and the Polyakov loop at $\mu=0$ is shown in Fig. 2. The meanfield results in the infinite volume limit is also shown as the 


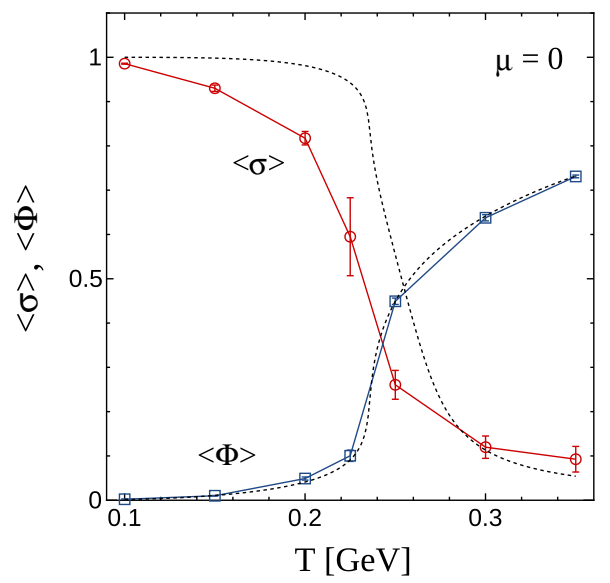

FIG. 2. The $T$-dependence of $\langle\sigma\rangle$ and $\langle\Phi\rangle$ at $\mu=0$ where $\langle\sigma\rangle$ is normalized by that at $T=\mu=0$ in the infinite volume limit. The dotted lines are mean-field results in the infinite volume limit as the eye guide. The difference between the eye guide and our Monte-Carlo result is nothing but the finite size effect which has been observed in Ref. [41].

eye guide. Because of the finite size effect, $\langle\sigma\rangle$ is deviated from the mean-field results in the infinite volume limit and this result is consistent with results obtained in Ref. [41]. In the present calculation, $V$ depends on $T$ and thus the finite size effect becomes serious when $T$ increases. The expectation values of $\vec{\pi}$ are consistent with zero in $2 \sigma$ error. At moderate $T$, the error bar becomes large because of the spreading behavior of the configuration which may come from the chiral properties. By increasing the configuration number, we will make it small.

The $\mu$-dependence of the average phase factor on the original integral-path at $T=100 \mathrm{MeV}$ and $\mu=300 \mathrm{MeV}$ is shown in Fig. 3. We also show the results after twice optimization in the figure. It can be clearly seen that $\left\langle e^{i \theta}\right\rangle$

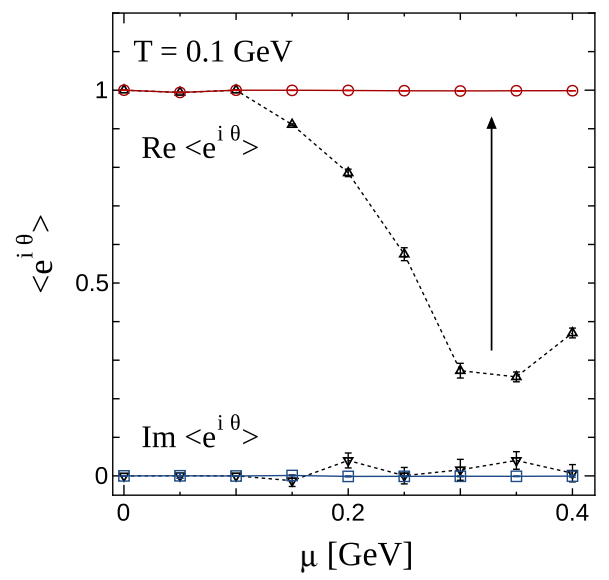

FIG. 3. The $\mu$-dependence of $\left\langle e^{i \theta}\right\rangle$ at $T=100 \mathrm{MeV}$ with $k=64$. The dotted and solid lines indicate results on the original and modified integral paths, respectively. The modified integral path is obtained after twice optimization.

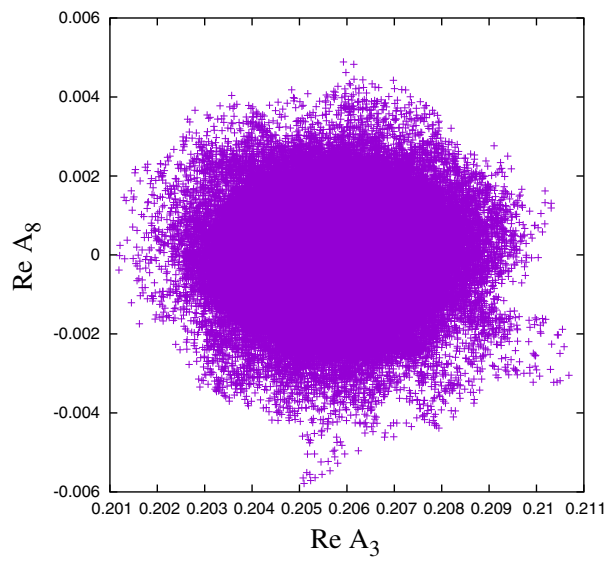

FIG. 4. The scatter plot of $\operatorname{Re} A_{3}$ and $\operatorname{Re} A_{8}$ at $T=100 \mathrm{MeV}$ and $\mu=300 \mathrm{MeV}$. We here use 80000 configurations.

becomes small around the chiral transition region, $T \sim 335 \mathrm{MeV}$, and then the model sign problem seriously appears. In the present computation, we use $k=64$ and thus $\left\langle e^{i \theta}\right\rangle$ does not become small so much, but it will be worse when we consider larger $k$ because $\left\langle e^{i \theta}\right\rangle$ is exponentially suppressed by $k$. Because of this problem, we need further investigation on the competition between the suppression from the system size and the improvement via the path optimization method in the average phase factor. It will be reported elsewhere.

For the reader's convenience, we show the scatter plot of $\operatorname{Re} A_{3}$ and $\operatorname{Re} A_{8}$ at $T=100 \mathrm{MeV}$ and $\mu=300 \mathrm{MeV}$ in Fig. 4. The spreading behavior of $\operatorname{Re} A_{3}$ is larger than that of $\operatorname{Re} A_{8}$ and then we can see the localized configurations.

To improve the average phase factor, we use the path optimization method. Figure 5 shows the average phase factor at each optimization step with $T=100 \mathrm{MeV}$ and $\mu=300 \mathrm{MeV}$ as an example. After one optimization step $\left(\mathrm{N}_{\mathrm{opt}}=1\right)$, the average phase factor becomes worse. Since

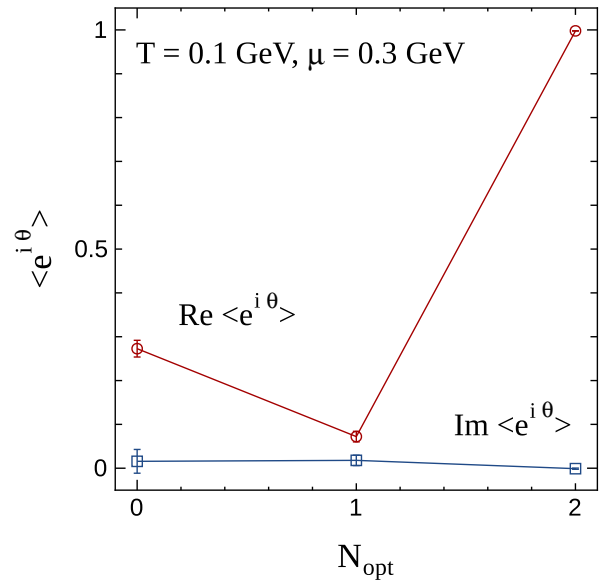

FIG. 5. The average phase factor at each optimization step with $T=100$ and $\mu=300 \mathrm{MeV}$. 

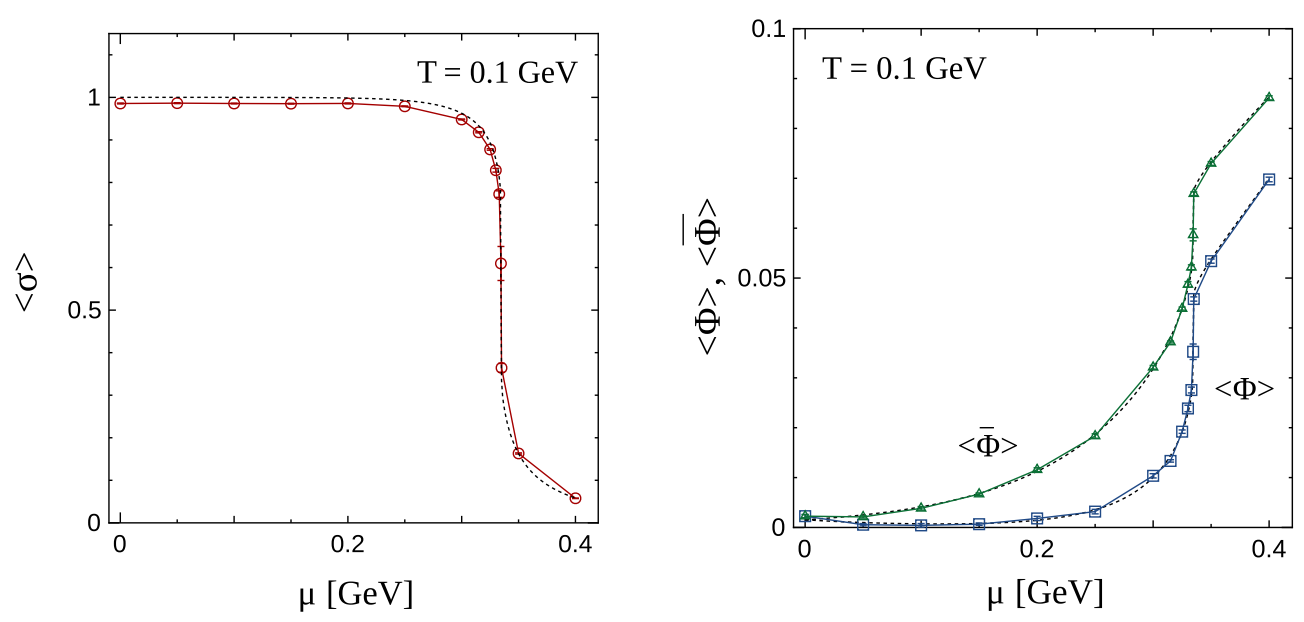

FIG. 6. The left (right) panel shows the $\mu$-dependence of $\langle\sigma\rangle(\langle\Phi\rangle)$ with $T=100$ and $\mu=300 \mathrm{MeV}$ where $\langle\sigma\rangle$ is normalized by that at $T=\mu=0$ in the infinite volume limit. The dotted lines are mean-field results with imposing the $\mathcal{C K}$ symmetry on the fermion determinant in the infinite volume limit as the eye guide.

the starting configurations used in the first optimization are generated on the original integral-path and thus those are expected to be located far from the relevant points of the optimized integral-path and then the average phase factor becomes worse temporally. However, the average phase factor is significantly improved after few optimization steps. In the optimization process, we employ widely used parameter set of the Adam algorithm and thus the step size of the optimization is expected to be not so large. The fast optimization is considered as follows: In the present calculation, the one optimization step has 8000 mini-batch training where parameters in the neural network are updated. Thus, the effective optimization step is large and then we can sufficiently improve the average phase factor with few $N_{\text {opt }}$. After the optimization, the probability distribution can be different in the original configurations and then the average phase factor once becomes worth. In the second optimization step, we adopt the configuration according to the optimized manifold and then the sufficient average phase factor is obtained. In the case at $T=$ $100 \mathrm{MeV}$ and $\mu=300 \mathrm{MeV}$ with $k=64, \mathrm{~N}_{\mathrm{opt}}=2$ are enough to make the average phase factor close to 1 . It should be noted that the present tendency of the improvement depends on several initial conditions of parameters and thus we should try with several set of parameters when the optimization does not work. Also, an increase of units in the hidden layer may lead to better convergence [57]. It should be noted that we must improve the numerical cost of the path optimization method in the future. The present method need at least $N_{\text {dof }}^{3}$ additional numerical cost where $N_{\text {dof }}=6$. With the lattice regularization, we will consider the system size also. To reduce the numerical cost, we will need some more extension of the path optimization method. One promising approach is the reduction of the Jacobian computation; the diagonal ansatz of the Jacobian [32] and the nearest-neighbor lattice-cites approximation [31] are examples.
The $\mu$-dependence of the chiral condensate and the Polyakov-loop after performing the path optimization are shown in Fig. 6. The mean-field results in the infinite volume with imposing the $\mathcal{C K}$ symmetry to the fermion determinant is also shown as the eye guide. Since there is the finite size effect, the eye guide and our Monte-Carlo results are deviated a little bit. Actually, if the shoulder of the action which appears between the chiral symmetry broken and restored points, the chiral condensate becomes smaller (bigger) than that at low (high) $T$ in the infinite volume limit. Therefore, the difference becomes small at high $\mu$ region because the shoulder becomes irrelevant. This tendency can be also seen from the $T$-dependence of the chiral condensate in Fig. 2 and Ref. [41]. We can correctly reproduce the relation $\langle\bar{\Phi}\rangle \neq$ $\langle\Phi\rangle$ with $\langle\bar{\Phi}\rangle,\langle\Phi\rangle \in \mathbb{R}$ in the PNJL model at finite $\mu$ by using the path optimization method. These results mean that the path optimization method can well work in the QCD effective model which has the model sign problem. Also, we can expect that the complexification of the temporal gluon field may be sufficient to control the sign problem in the lattice QCD with the path optimization method since the lattice QCD and the PNJL model share similar properties about the sign problem.

\section{SUMMARY}

In this article, we apply the path optimization method to the Polyakov-loop extended Nambu-Jona-Lasinio (PNJL) model to circumvent the model sign problem. This study is the first attempt to apply the path optimization method to the effective model with dynamical quarks based on QCD. The PNJL model can describe the chiral phase transition and also approximated confinement-deconfinement transition and thus it is a good starting point to investigate the 
QCD phase structure at finite real chemical potential $(\mu)$. Therefore, we choose it as the typical QCD effective model in this article.

The temporal components of the gluon field, $A_{3}$ and $A_{8}$, are complexified and the modified integral path in the complex space is expressed by using the feedforward neural network by minimizing the cost function which reflects the seriousness of the sign problem. Then, the sign problem becomes the optimization problem. Parameters in the feedforward neural network are optimized via the backpropagation method. The neural network tries to increase the average phase factor compared with the previous optimization step and thus it is nothing but the unsupervised learning. The scalar and pseudo-scalar auxiliary fields are treated as real valuables. This treatment is motivated by the Lefschetz thimble analysis done in Ref. [37]. In the actual optimization process, we use the minibatch training with the Adam algorithm.

We have shown that our treatment of variables of integration works well; the average phase factor is sufficiently improved after the optimization at finite $\mu$. This means that the path optimization method can resolve the model sign problem based on the hybrid Monte Carlo method. In this study, we use the homogeneous ansatz for the auxiliary fields and thus we cannot go beyond the mean-field approximation, but it is a first step to correctly treat the sign problem in the QCD effective models. By considering the straightforward extension of the present formulation, we will go beyond the mean-field approximation of the QCD effective models. We leave the actual simulation as our future work. From these results, we may expect that the complexification of the temporal gluon fields is the sufficient way to control the sign problem in the lattice QCD with the path optimization method because QCD and the PNJL model share similar properties about the sign problem.

\section{ACKNOWLEDGMENTS}

This work is supported in part by the Grants-inAid for Scientific Research from JSPS (No. 15K05079, No. 15H03663, No. 16K05350, No. 18K03618), the Grants-in-Aid for Scientific Research on Innovative Areas from MEXT (No. 24105001, No. 24105008), and by the Yukawa International Program for Quark-hadron Sciences (YIPQS).
[1] O. Miyamura, S. Choe, Y. Liu, T. Takaishi, and A. Nakamura, Phys. Rev. D 66, 077502 (2002).

[2] C. R. Allton, M. Doring, S. Ejiri, S. J. Hands, O. Kaczmarek, F. Karsch, E. Laermann, and K. Redlich, Phys. Rev. D 71, 054508 (2005).

[3] R. Gavai and S. Gupta, Phys. Rev. D 78, 114503 (2008).

[4] Z. Fodor and S. Katz, Phys. Lett. B 534, 87 (2002); J. High Energy Phys. 03 (2002) 014; 04 (2004) 050.

[5] Z. Fodor, S. Katz, and K. Szabo, Phys. Lett. B 568, 73 (2003).

[6] P. de Forcrand and O. Philipsen, Nucl. Phys. B642, 290 (2002); B673, 170 (2003).

[7] M. D'Elia and M.-P. Lombardo, Phys. Rev. D 67, 014505 (2003); 70, 074509 (2004).

[8] H.-S. Chen and X.-Q. Luo, Phys. Rev. D 72, 034504 (2005).

[9] A. Hasenfratz and D. Toussaint, Nucl. Phys. B371, 539 (1992).

[10] A. Alexandru, M. Faber, I. Horvath, and K.-F. Liu, Phys. Rev. D 72, 114513 (2005).

[11] S. Kratochvila and P. de Forcrand, Phys. Rev. D 73, 114512 (2006).

[12] P. de Forcrand and S. Kratochvila, Nucl. Phys. B, Proc. Suppl. 153, 62 (2006).

[13] A. Li, A. Alexandru, K.-F. Liu, and X. Meng, Phys. Rev. D 82, 054502 (2010).

[14] P. de Forcrand, Proc. Sci., LATTICE2009 (2009) 010.

[15] G. Parisi and Y.-s. Wu, Sci. Sin. 24, 483 (1981).

[16] G. Parisi, Phys. Lett. 131B, 393 (1983).

[17] E. Witten, AMS/IP Stud. Adv. Math. 50, 347 (2011).
[18] M. Cristoforetti, F. Di Renzo, and L. Scorzato (AuroraScience Collaboration), Phys. Rev. D 86, 074506 (2012).

[19] H. Fujii, D. Honda, M. Kato, Y. Kikukawa, S. Komatsu, and T. Sano, J. High Energy Phys. 10 (2013) 147.

[20] G. Aarts, E. Seiler, and I.-O. Stamatescu, Phys. Rev. D 81, 054508 (2010).

[21] J. Nishimura and S. Shimasaki, Phys. Rev. D 92, 011501 (2015).

[22] Y. Mori, K. Kashiwa, and A. Ohnishi, Phys. Lett. B 781, 688 (2018).

[23] J. Nishimura and S. Shimasaki, EPJ Web Conf. 175, 07018 (2018).

[24] Y. Ito and J. Nishimura, J. High Energy Phys. 12 (2016) 009.

[25] F. Di Renzo and G. Eruzzi, Proc. Sci., LATTICE2016 (2016) 047 .

[26] F. Di Renzo and G. Eruzzi, Phys. Rev. D 97, 014503 (2018).

[27] F. Attanasio and B. Jaeger, arXiv:1808.04400.

[28] Y. Mori, K. Kashiwa, and A. Ohnishi, Phys. Rev. D 96, 111501 (2017).

[29] Y. Mori, K. Kashiwa, and A. Ohnishi, Prog. Theor. Exp. Phys. 2018, 023 B04 (2018).

[30] A. Ohnishi, Y. Mori, and K. Kashiwa, EPJ Web Conf. 175, 07043 (2018).

[31] F. Bursa and M. Kroyter, J. High Energy Phys. 12 (2018) 054.

[32] A. Alexandru, P. F. Bedaque, H. Lamm, and S. Lawrence, Phys. Rev. D 97, 094510 (2018). 
[33] P. N. Meisinger, T. R. Miller, and M. C. Ogilvie, Phys. Rev. D 65, 034009 (2002).

[34] K. Fukushima, Phys. Lett. B 591, 277 (2004).

[35] C. Ratti, M. A. Thaler, and W. Weise, Phys. Rev. D 73, 014019 (2006).

[36] K. Fukushima and Y. Hidaka, Phys. Rev. D 75, 036002 (2007).

[37] Y. Tanizaki, H. Nishimura, and K. Kashiwa, Phys. Rev. D 91, 101701 (2015).

[38] K. Kashiwa and A. Ohnishi, Phys. Lett. B 750, 282 (2015).

[39] K. Kashiwa and A. Ohnishi, Phys. Rev. D 93, 116002 (2016).

[40] K. Kashiwa and A. Ohnishi, Phys. Lett. B 772, 669 (2017).

[41] M. Cristoforetti, T. Hell, B. Klein, and W. Weise, Phys. Rev. D 81, 114017 (2010).

[42] S. Roessner, C. Ratti, and W. Weise, Phys. Rev. D 75, 034007 (2007).

[43] K. Kashiwa, H. Kouno, T. Sakaguchi, M. Matsuzaki, and M. Yahiro, Phys. Lett. B 647, 446 (2007).

[44] G. Cybenko, Math. Control Signals Syst. 2, 303 (1989).

[45] K. Hornik, Neural Netw. 4, 251 (1991).

[46] D. E. Rumelhart, G. E. Hinton, R. J. Williams et al., Cognit. model. 5, 1 (1988).
[47] H. Nishimura, M. C. Ogilvie, and K. Pangeni, Phys. Rev. D 90, 045039 (2014).

[48] H. Nishimura, M. C. Ogilvie, and K. Pangeni, Phys. Rev. D 91, 054004 (2015).

[49] S. Roessner, T. Hell, C. Ratti, and W. Weise, Nucl. Phys. A814, 118 (2008).

[50] A. Alexandru, P. F. Bedaque, H. Lamm, and S. Lawrence, Phys. Rev. D 96, 094505 (2017).

[51] A. Alexandru, G. Basar, P. F. Bedaque, G. W. Ridgway, and N.C. Warrington, J. High Energy Phys. 05 (2016) 053.

[52] M. Fukuma and N. Umeda, Prog. Theor. Exp. Phys. 2017, 073B01 (2017).

[53] A. Alexandru, G. Basar, P.F. Bedaque, and N.C. Warrington, Phys. Rev. D 96, 034513 (2017).

[54] D. Kingma and J. Ba, arXiv:1412.6980.

[55] J. Duchi, E. Hazan, and Y. Singer, J. Mach. Learn. Res. 12, 2121 (2011).

[56] X. Glorot and Y. Bengio, in Proceedings of the Thirteenth International Conference on Artificial Intelligence and Statistics (2010), pp. 249-256.

[57] J. Frankle and M. Carbin, arXiv:1803.03635. 